\title{
On the nature of the lightest scalar resonances ${ }^{1}$
}

\author{
Z. X. Sun, L. Y. Xiao, Z. G. Xiao, H. Q. Zheng and Z. Y. Zhou \\ Department of Physics, Peking University, Beijing 100871, P. R. China
}

\begin{abstract}
We briefly review the recent progresses in the new unitarization approach being developed by us. Especially we discuss the large $N_{c} \pi \pi$ scatterings by making use of the partial wave $S$ matrix parametrization form. We find that the $\sigma$ pole may move to the negative real axis on the second sheet of the complex $s$ plane, therefore it raises the interesting question that this ' $\sigma$ ' pole may be related to the $\sigma$ in the linear $\sigma$ model.
\end{abstract}

The problem of how to restore unitarity and meanwhile respecting chiral perturbation amplitudes at low energies is very interesting and also difficult. A simple solution one has when dealing with such a difficult problem is the Padé approximation and its variations, which achieved some phenomenological success. Nevertheless, the Padé approximation encounters serious problems [1] which can hardly be resolved within the method itself. For this reason, it is worthwhile to make further efforts to study the problem from a more rigorous and different point of view.

In Refs. [2, 3, 4], a new parametrization form - which we call as the 'PKU' parametrization form - for partial wave $S$ matrices in the elastic channel is developed, which, when combined with chiral symmetry, has been proven useful in probing the resonance structure of low energy strong interaction dynamics. For example, it reveals that the existence of the $\sigma[5,4]$ meson is fully consistent with chiral symmetry. Combining with crossing symmetry, it further predicts the $\sigma$ pole mass and width to be $M_{\sigma}=470 \pm 50 \mathrm{MeV}, \Gamma_{\sigma}=570 \pm 50 \mathrm{MeV}$. [4] Also it is shown that there should exist the $\kappa$ resonance if the $\pi K$ scattering length in the $\mathrm{I}, \mathrm{J}=1 / 2,0$ channel does not deviate much from the value predicted by chiral perturbation theory. [3] The PKU parametrization form is the following,

$$
S^{\text {phy. }}=\prod_{i} S_{i}^{\text {poles }} \cdot S^{\text {cut }}
$$

where $S_{i}^{\text {poles }}$ denote various kinds of poles: resonance, bound state and virtual bound state. For resonance poles we have

$$
S^{R}(s)=\frac{M^{2}\left(z_{0}\right)-s+i \rho(s) s G\left[z_{0}\right]}{M^{2}\left(z_{0}\right)-s-i \rho(s) s G\left[z_{0}\right]},
$$

\footnotetext{
1 Talk presented by Zheng at "Quark Confinement and Hadron Spectroscopy VI", 21-25 Sept. 2004, Cagliari, Italy
} 
where

$$
M^{2}\left(z_{0}\right)=\operatorname{Re}\left[z_{0}\right]+\frac{\operatorname{Im}\left[z_{0}\right] \operatorname{Im}\left[z_{0} \rho\left(z_{0}\right)\right]}{\operatorname{Re}\left[z_{0} \rho\left(z_{0}\right)\right]}, G\left[z_{0}\right]=\frac{\operatorname{Im}\left[z_{0}\right]}{\operatorname{Re}\left[z_{0} \rho\left(z_{0}\right)\right]},
$$

where $z_{0}$ denotes the resonance pole location on the complex $s$ plane. The Eq. (2) is very interesting as it reveals the remarkable difference between a narrow resonance located far above the threshold and a light and broad resonance. In fact, $s=M^{2}\left(z_{0}\right)$ is the place where the resonance's contribution to the phase shift passes $\pi / 2$. However, a light and broad resonance may correspond to a very large $M^{2}\left(z_{0}\right)$. The Eq. (2) for a light and broad pole actually nicely summarizes the major contribution to $I J=00$ channel $\pi \pi$ scattering phase shift at low energies. The $S^{c u t}$ in Eq. (1) no longer contains any pole and for $\pi \pi$ scatterings it can be parameterized as:

$$
S^{c u t}=e^{2 i \rho f(s)}, \quad f(s)=\frac{s}{\pi} \int_{L} \frac{\operatorname{Im}_{L} f\left(s^{\prime}\right)}{s^{\prime}\left(s^{\prime}-s\right)}+\frac{s}{\pi} \int_{R} \frac{\operatorname{Im}_{R} f\left(s^{\prime}\right)}{s^{\prime}\left(s^{\prime}-s\right)} .
$$

where $L=(-\infty, 0]$ and $R$ denotes physical cuts higher than the $2 \pi$ cut, and $\operatorname{Im}_{L, R} f=$ $-\frac{1}{2 \rho} \log \left|S^{p h y}\right|$. Before proceeding it should be emphasized that the above parametrization form is only obtainable by assuming analyticity on the whole cut plane, which can be derived from Mandelstam representation but nevertheless not proven rigorously from field theory. However the Lehman-Martin domain of analyticity is large enough for phenomenological applications. Therefore the parametrization form described above may afford a good approximation to the real situation.

At low energies one may approximate $S^{p h y}$ appeared in the dispersion integral by $S^{\chi \mathrm{PT}}$ on $L$, to estimate the background contributions from the left after introducing a proper cutoff parameter to truncate the dispersion integral. One may then get more information from the parametrization form discussed above. Rewrite Eq. (1) as

$$
\prod_{i} S_{i}^{\text {poles }}=S^{\text {phy. }}\left(S^{c u t}\right)^{-1}
$$

as stated before the r.h.s. of the above equation can be expressed by low energy quantities appeared in, for example, the $O\left(p^{4}\right)$ low energy chiral Lagrangian. Expanding both sides of Eq. (5) at threshold, one relates the pole parameters to the low energy constants of the effective Lagrangian. Making use of the $N_{c}$ counting rule of low energy constants [6] one can thus trace how the pole moves on the complex $s$ plane when $N_{c}$ varies. Nevertheless, in order to get the pole trajectory we need some further assumptions. Precisely we assume one pole dominates the l.h.s. of Eq. (5) for arbitrarily value of $N_{c}$. Such an assumption is of course only a speculation and may be subjected to criticism, though in the case of $N_{c}=3$ one pole dominance at low energies is a good approximation. [4] Nevertheless we will proceed with this working assumption to see what happens. Here we keep all the $N_{c}$ dependence including chiral logs. We make use of $O\left(p^{4}\right) \chi \mathrm{PT}$ results to approximate $S^{p h y}$. and to calculate $f(s)$, and neglect the right hand cut integral and truncated the left hand integral at certain value. In this way we get for $N_{c}=3$ the pole mass of $\sigma$ in rather good agreement with more realistic calculations. The $N_{c}$ dependence of the pole mass can be traced numerically. The result is shown in Fig. 1 

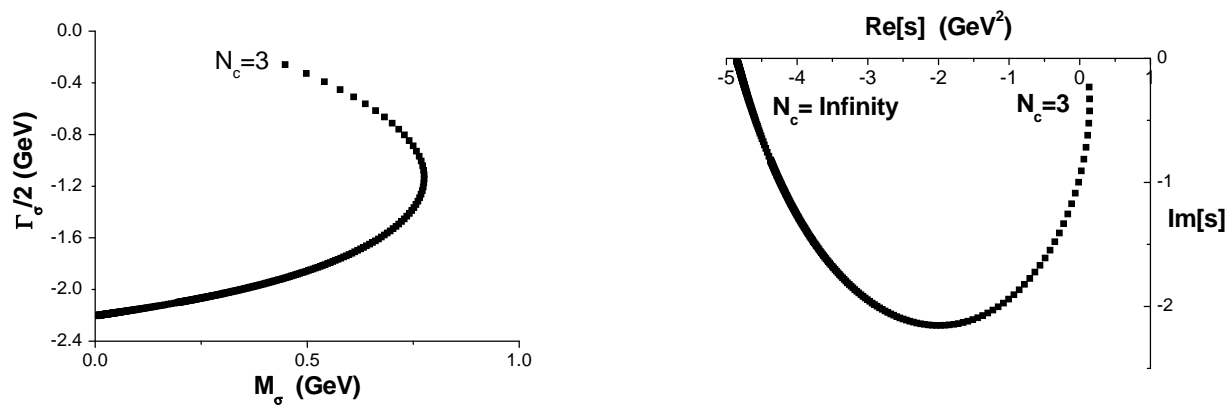

FIGURE 1. The trajectory of the $\sigma$ pole: left) the complex $\sqrt{s}$ plane; right) the complex $s$ plane.

and it is amazing to see that the pole moves to the negative real axis when $N_{c}$ approaches infinity. If the pole location is plotted for large but finite value of $N_{c}$ on the $E$ plane, it will stay somewhere on the complex plane. The latter is the observation made in Ref. [7] However, the pole trajectory on the $s$ plane clearly indicates what is the correct interpretation: the mass square becomes negative when the pole moves towards the real negative axis, rather than that the $\sigma$ resonance has a large width of $O(1)$. Many phenomenological studies predicted that the $\sigma$ pole moves towards left, though some finally touch the negative real axis, some do not. [8]. However it should be pointed out that the $\sigma$ pole trajectory is very flexible. In our scheme, it is actually easy to tune the $L_{i}$ parameters within $1 \sigma$ error bar to convert the pole position to move towards the real positive $s$ axis above the threshold. We have checked that such a flexibility also exists in the Padé amplitudes. Therefore our present scheme has a similar prediction on the pole trajectory comparing with the Padé amplitude. Therefore only one definite conclusion can be made by us: the $\sigma$ pole moves to the real axis in the large $N_{c}$ limit, and $M^{2} \sim O(1), G \sim O\left(1 / N_{c}\right)$ (more details will appear soon). Finally we remark that a resonance with negative mass square, though seems odd, does not seem to spoil any fundamental principles because it locates on the second sheet. If the ' $\sigma$ ' discussed in this paper really gets a negative $M^{2}$, one wonders wether it has anything to do with the $\sigma$ in the linear $\sigma$ model.

Acknowledgment: We would like to thank Frieder Kleefeld and George Rupp for helpful discussions.

\section{REFERENCES}

1. Q. Ang et al, Commun. Theor. Phys. 36(2001)563; G. Y. Qin eta al, Phys.Lett.B542:89-99,2002

2. H. Q. Zheng, hep-ph/0304173, J. Y. He, Z. G. Xiao and H. Q. Zheng, Phys. Lett. B526(2002)59 (Erratum: ibid. B549(2002)362).

3. H. Q. Zheng et al., Nucl. Phys. A733(2004)235.

4. Z. Y. Zhou et al, hep-ph/0406271 v2.

5. Z. G. Xiao and H. Q. Zheng, Nucl. Phys. A695(2001)273.

6. J. Gasser and H. Leutwyler, Nucl. Phys. B250(1985)465.

7. J. R. Palaez, Phys. Rev. Lett. 92(2004)1020001.

8. V. E. Markushin and M. P. Locher, in Frascati 1999, Hadron spectroscopy 229-236 (hep-ph/9906246); E. Van Beveren and G. Rupp, Eur. Phys. J. C22 (2001) 493. 